\title{
Radiofrequency Interference Identified
}

National Cancer Institute

\section{Source}

National Cancer Institute. Radiofrequency Interference Identified. NCI Thesaurus. Code C92101.

Problems due to radiofrequency interference. RFI is a disturbance that affects an electrical circuit due to either electromagnetic conduction or electromagnetic radiation emitted from an external source. 